\title{
Mixed-State Thermodynamics of Superconductors with Moderately Large Paramagnetic Effects
}

\author{
Hiroto ADACHI *, Masanori ICHIOKA and Kazushige MACHIDA \\ Department of Physics, Okayama University, Okayama 700-8530
}

\begin{abstract}
Effects of Pauli paramagnetism on thermodynamic quantities in a vortex state, such as the specific heat $C$ and magnetization $M$, are studied using the quasiclassical Eilenberger formalism. We demonstrate that with an increase of paramagnetic depairing effect, the sigh of the curvature of the field dependence of $C$ changes from negative to positive, and that the Maki parameter $\kappa_{2}$ becomes an increasing function of temperature. Our results provide a natural explanation for the unusual field dependence of $C$ seen in $\mathrm{CeCoIn}_{5}$ in terms of the paramagnetic effect.
\end{abstract}

KEYWORDS: mixed state, thermodynamic quantity, paramagnetic effect, CeColn 5

There has been much attention focused on heavy fermion superconductors, in particular on the newly found $\mathrm{CeCoIn}_{5} .{ }^{1}$ This material with a tetragonal symmetry exhibits a first-order transition ${ }^{2-5}$ at $H_{c 2}$ both for $\mathbf{H} \| c$ and $\mathbf{H} \perp c$. In a high field region for $\mathbf{H} \perp c$, the possible existence of the Fulde-Ferrell-LarkinOvchinnikov (FFLO) state ${ }^{6-8}$ is highly debated currently, because several experiments strongly indicate that the Pauli paramagnetic effect causes the first-order transition, which may lead to the FFLO state. The pairing symmetry of this material on either $d_{x y}{ }^{9,10}$ or $d_{x^{2}-y^{2}}{ }^{2}$ is also controversial. One of the puzzling features concerning the pairing is that the specific heat $C^{9,11,12}$ obeys neither the so-called Volovik effect ${ }^{13} C \propto \sqrt{B}$ expected for a line node case nor $C \propto B$ for a gapful case, ${ }^{14-16}$ where $B$ is the spatially averaged internal field. Instead $\mathrm{CeCoIn}_{5}$ shows a $C \propto B^{2}$-like behavior. We point out that a similar behavior in $C(B)$ is observed also in $\mathrm{UBe}_{13} \cdot{ }^{17} \mathrm{It}$ is naively expected that the exponent $\eta$ in $C \propto B^{\eta}$ must be $\eta \leq 1$, because each vortex carries a certain zero-energy density of states and $C / C_{\mathrm{n}} \geq B / H_{c 2}$, where $C_{\mathrm{n}}$ is the normal state specific heat. Thus, the observed $C \propto B^{2}$ like behavior is highly unusual and a puzzling issue to be solved.

In a clean singlet type-II superconductor under a magnetic field, there are two microscopic energy scales ${ }^{18-20}$ for the depairing effects: the Zeeman energy $\mu B$ for the paramagnetic depairing and the orbital depairing energy $\mathcal{E}_{B}=v_{F} / r_{B}$ associated with the Doppler shift, where $v_{F}$ is a Fermi velocity, $r_{B}=\sqrt{\phi_{0} / 2 \pi B}$ with $\phi_{0}$ being the flux quantum. In materials with a small Fermi velocity such as heavy fermion superconductors, the role of the Zeeman energy compared to $\mathcal{E}_{B}$ cannot be neglected when discussing the thermodynamic properties. As for the phenomena near $H_{c 2}$, the effects of Pauli paramagnetism on the mixed state have been studied by Adachi and Ikeda using the nonlocal Ginzburg-Landau (GL) theory. ${ }^{5}$ To describe the thermodynamic quantities in lower fields we use the quasiclassical Eilenberger formalism. ${ }^{21}$

In this Letter, we clarify how Pauli paramagnetism affects the mixed-state thermodynamics using the quasiclassical Eilenberger formalism. Using the approximate method given in ref. 22 , we demonstrate that in a superconductor with moderately large paramagnetic effects under a magnetic field, the specific heat varies roughly quadratically with the field, $C \propto B^{2}$, consistent with the experimental data in $\mathrm{CeCoIn}_{5} \cdot{ }^{9,11,12}$ At a lower temperature with a stronger paramagnetic effect, our result shows a magnetization jump at $H_{c 2}$, which is also observed in $\mathrm{CeCoIn}_{5} .{ }^{4}$ We give a simple thermodynamic argument to relate the positive curvature of $C(B)$ to the magnetization behavior under the paramagnetic effect, giving rise to the internal consistency between these quantities. Near $H_{c 2}$, our numerical solution coincides, of course, with the results of the nonlocal GL theory given in ref. 5 .

First, let us briefly introduce our approach. We start with the Eilenberger equation ${ }^{21}$ incorporating the paramagnetic effect ${ }^{23,24}\left(\hbar=k_{B}=1\right)$;

$$
\left(2 \varepsilon_{\mu}+\mathrm{i} \mathbf{v} \cdot \boldsymbol{\Pi}\right) f\left(\varepsilon_{\mu}, \mathbf{p}, \mathbf{r}\right)=2 g\left(\varepsilon_{\mu}, \mathbf{p}, \mathbf{r}\right) w_{\mathbf{p}} \Delta(\mathbf{r}),
$$

where $f\left(\varepsilon_{\mu}, \mathbf{p}, \mathbf{r}\right), f^{\dagger}\left(\varepsilon_{\mu}, \mathbf{p}, \mathbf{r}\right)=\left[f\left(\varepsilon_{\mu}^{*},-\mathbf{p}, \mathbf{r}\right)\right]^{*}$, and $g\left(\varepsilon_{\mu}, \mathbf{p}, \mathbf{r}\right)=\sqrt{1-f f^{\dagger}}$ are the quasiclassical Green's functions. Here, $\mathbf{v}=v_{F} \hat{\mathbf{p}}$ is a Fermi velocity, $\boldsymbol{\Pi}=$ $-\mathrm{i} \nabla+\left(2 \pi / \Phi_{0}\right) \mathbf{A}$ is a gauge invariant gradient, $T_{c}$ is a transition temperature at a zero field. The fermionic Matsubara frequency $\varepsilon_{n}=2 \pi T(n+1 / 2)$ combined with the (renormalized) Zeeman energy is denoted as $\varepsilon_{\mu}=\varepsilon_{n}-\mathrm{i} \mu B$, and the gap function is expressed as $\Delta_{\mathbf{p}}(\mathbf{r})=w_{\mathbf{p}} \Delta(\mathbf{r})$ with the pairing function $w_{\mathbf{p}}$ and the pair field $\Delta(\mathbf{r})$. In the following, the pairing state of a $d$-wave $\left(w_{\mathbf{p}}=2 \sqrt{2} \hat{p}_{x} \hat{p}_{y}\right)$ or $s$-wave $\left(w_{\mathbf{p}}=1\right)$ is assumed. Since the detailed shape of the Fermi surface does not change our main results essentially, we consider a quasitwo-dimensional isotropic Fermi surface. In the mixed state containing field-induced vortices, the pair field $\Delta$ can be expanded into each Landau level:

$$
\Delta=\Delta_{0} \sum_{N=0}^{N_{\max }} d_{N} \psi_{N},
$$


where $\psi_{N}=\sum_{m=-\infty}^{\infty} C_{m} \frac{H_{N}(y+\nu m)}{\sqrt{2^{N} N !}} \mathrm{e}^{-(y+\nu m)^{2} / 2-\mathrm{i} \nu m x}$, $\Delta_{0}=1.764 T_{c}, C_{m}=\left(\nu^{2} / \pi\right)^{1 / 4} \mathrm{e}^{-\mathrm{i} \pi \zeta m^{2}}, H_{N}$ the $N$-th Hermite polynomial, and the lengths are measured in units of $r_{B}$. The real constants $\zeta$ and $\nu$ are set to be $\zeta=1 / 2$ and $\nu=\left(3 \pi^{2}\right)^{1 / 4}$, assuming a triangular vortex lattice. The difference in vortex configuration gives minor contributions. To solve eq. (1), we adopt the approximate method given in ref. 22 , which gives

$$
f=2 g w_{\mathbf{p}} \int_{0}^{\infty} d \rho \mathrm{e}^{-2 \varepsilon_{\mu} \rho}\left(\Delta_{0} \sum_{N=0}^{N_{\max }} \alpha_{N} d_{N}\right) .
$$

Here, the quantity $\alpha_{N}$ is given by $\alpha_{N}=$ $\sum_{m} C_{m} \frac{H_{N}(y+\nu m-\operatorname{Re} \lambda)}{\sqrt{2^{N} N !}} \mathrm{e}^{-\left(|\lambda|^{2}-\lambda^{2}\right) / 4} \mathrm{e}^{-(y+\nu m-\lambda)^{2} / 2-\mathrm{i} \nu m x}$, and $\lambda=\left(v_{y}+\mathrm{i} v_{x}\right) \rho / r_{B}$. The above procedure uses an approximation similar to that used by Pesch, ${ }^{25}$ in which the operator $\boldsymbol{\Pi}$ does not pick up the spatial variation in $g$, but only that in $\Delta$. For extreme type-II materials with a large GL parameter $\kappa$ (in $\left.\mathrm{CeCoIn}_{5} \kappa \gg 10\right)$, this approximation provides a fairly good description for almost all experimentally relevant fields. Note that in contrast to the method by Pesch, our method can reproduce the first non-Gaussian term of the nonlocal GL free energy. ${ }^{5}$ Recently, a simplified version of our method has been widely used $^{26}$ in slightly different contexts, in which the spatial degree of freedom is integrated out in advance.

The expansion coefficients $\left\{d_{N}\right\}$ are determined by the following gap equation

$$
\left[\ln \left(\frac{T}{T_{c}}\right)+\sum_{n \geq 0} \frac{2 \pi T}{\varepsilon_{n}}\right] d_{N}=\frac{\pi T}{\Delta_{0}} \sum_{n \geq 0} \overline{\psi_{N}^{*}\left\langle w_{\mathbf{p}}^{*}\left(f+\left(f^{\dagger}\right)^{*}\right)\right\rangle},
$$

where $\langle\cdots\rangle$ and $\cdots$ indicate the Fermi surface and spatial average, respectively. The strength of the paramagnetic effect in this Letter is measured using $\alpha_{\text {para }} \equiv$ $\mu H_{\text {orb }} / 2 \pi T_{c}$, where $H_{\text {orb }}=0.561 \Phi_{0} / 2 \pi \xi_{0}^{2}$ is the twodimensional orbital limiting field and $\xi_{0}=v_{F} / 2 \pi T_{c}$. If we define another parameter introduced by Maki as $\alpha_{\mathrm{M}}=\sqrt{2} H_{\mathrm{orb}} / H_{P}\left(H_{P}=\Delta_{0} / \sqrt{2}\right),{ }^{18,27}$ these two parameters are related as $\alpha_{\mathrm{M}}=7.12 \alpha_{\text {para }}$. Throughout this Letter, we limit our discussion to the parameter region $\alpha_{\text {para }} \leq 0.6$ so that the possibility of either the vortex state formed by odd Landau levels ${ }^{10,24,28}$ or the FFLO vortex state ${ }^{10}$ is safely excluded, and we consider only the lowest Landau level. Furthermore, we define $\kappa$ as $\kappa^{-2}=4 \pi N(0) \Delta_{0}^{2} / H_{\text {orb }}^{2}$, where $N(0)$ is the density of states at the Fermi surface.

To confirm whether our method can correctly capture the paramagnetic effect in the mixed state, we first study the magnetization. In the presence of the paramagnetic effect, the mixed-state magnetization is decomposed into the diamagnetic part $M_{\text {dia }}$ and the paramagnetic part $M_{\text {para }}: 23,24$

$$
\begin{aligned}
4 \pi M_{\text {dia }}=-\frac{2 \pi^{2} N(0)}{B} T & \sum_{n \geq 0}\left\langle\frac{\overline{g\left(f^{\dagger}\left(w_{\mathbf{p}} \Delta\right)+f\left(w_{\mathbf{p}} \Delta\right)^{*}\right)}}{1+g}\right. \\
& \left.-2 \varepsilon_{n} \overline{(1-g)}\right\rangle+ \text { c.c., }
\end{aligned}
$$

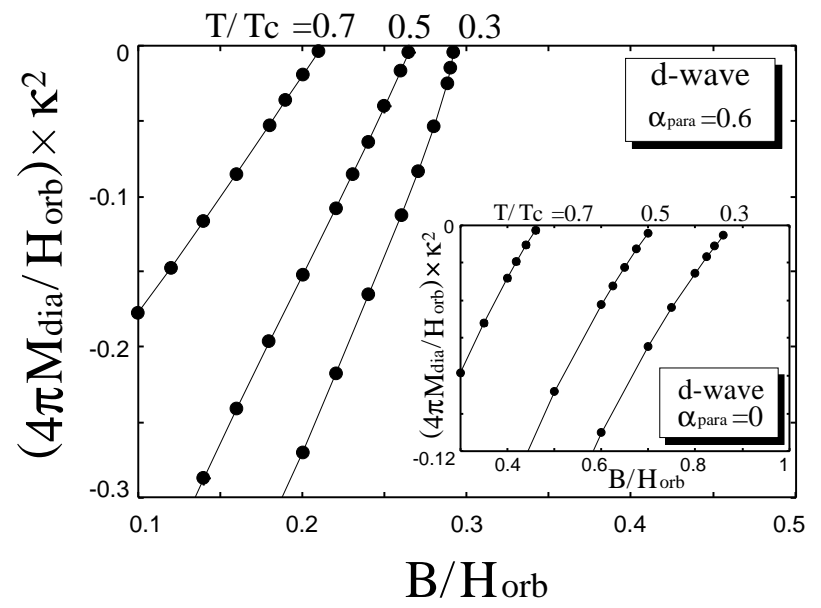

Fig. 1. Field dependence of $M_{\text {dia }}$ in $d$-wave superconductor with $\alpha_{\text {para }}=0.6$. Inset: corresponding data for $\alpha_{\text {para }}=0$.

$4 \pi M_{\text {para }}=4 \pi M_{\mathrm{n}}\left(1+\left(\frac{2 \pi T}{\mu B}\right) \operatorname{Im} \sum_{n \geq 0} \overline{\langle g\rangle}\right)$,

where $M_{\mathrm{n}}=2 \pi \mu^{2} N(0) B$ is the Pauli paramagnetism in the normal state. In the clean limit without paramagnetism, the Maki parameter $\kappa_{2}=$ $\left.\sqrt{\left[2 \beta_{A} d\left(4 \pi M_{\text {dia }}\right) / d H\right]^{-1}+0.5}\right|_{H c 2}$ with $\beta_{A}=1.1596$ is known to be a decreasing function of the temperature $T{ }^{29,30}$ In the high- $\kappa$ case, we approximately have $\kappa_{2} \propto$ $\left[\frac{d}{d B}\left(4 \pi M_{\mathrm{dia}}\right)\right]_{B=H_{c 2}}^{-1 / 2}$, and the magnetization slope at $H_{c 2}$ gives a rough estimate of $\kappa_{2}$. The inset of Fig. 1 shows the field dependences of $M_{\text {dia }}$ in a $d$-wave superconductor without the paramagnetic effect $\left(\alpha_{\text {para }}=0\right)$. We can see that $\kappa_{2}$ is a slowly decreasing function of $T$ in agreement with the results shown in refs. 29 and 30. Corresponding data with the paramagnetic effect $\left(\alpha_{\text {para }}=0.6\right)$ are shown in the main panel of Fig. 1. Contrary to the purely diamagnetic case, $\kappa_{2}$ in this case is an increasing function of $T$ consistent with the observation in $\mathrm{CeCoIn}_{5} \cdot{ }^{11,12}$ Our result should be compared with that of the dirtylimit analysis, ${ }^{27}$ where $\kappa_{2}$ was shown to be an increasing function of $T$ due to the paramagnetic effect.

Next, we study the magnetization at a lower temperature where a first-order transition at $H_{c 2}$ occurs. $^{5,28}$ Figure 2 shows the field dependences of $M_{\text {dia }}$ at $T / T_{c}=0.2$ in a $d$-wave superconductor. In a low field region, the paramagnetic effect is irrelevant and all the data for a different $\alpha_{\text {para }}$ value merge into a single curve. On the other hand, the slope $d M_{\mathrm{dia}} / d B$ increases with increasing $\alpha_{\text {para }}$ near $H_{c 2}$, and for $\alpha_{\text {para }}=0.6$, we find a discontinuous jump in magnetization at $H_{c 2}$ reflecting the first-order transition within the mean-field approximation. Indeed, if we plot the free energy against the pair field strength near $H_{c 2}$ (inset of Fig. 2), the free energy has a minimum at a nonzero value of $\left(\overline{|\Delta|^{2}}\right)^{1 / 2}$, implying a discontinuous jump in corresponding thermodynamic quantities. This explains the nearly discontinuous transition at $H_{c 2}$ observed in $\mathrm{CeCoIn}_{5} \cdot{ }^{2-4}$ We note here that we have also obtained essentially the same results also for the $s$-wave case.

Now, we consider the specific heat $C$. To obtain $C$ 
numerically, we first calculate the entropy difference between the normal and mixed states using ${ }^{23}$

$$
\begin{aligned}
\frac{S}{V}-\frac{S_{\mathbf{n}}}{V}=\frac{N(0)}{T}( & -\overline{|\Delta|^{2}}+\operatorname{Re} 2 \pi T \sum_{n \geq 0}\left\langle 2 \varepsilon_{n} \overline{(g-1)}\right. \\
& \left.\left.+\frac{\overline{f^{\dagger}\left(w_{\mathbf{p}} \Delta\right)+f\left(w_{\mathbf{p}} \Delta\right)^{*}}}{1+g}\right\rangle\right), \quad(7)
\end{aligned}
$$

where $S_{\mathrm{n}} / V=2 \pi^{2} N(0) T / 3$ is the entropy in the normal state. Then we perform a polynomial interpolation through the numerical data, and obtain $C$ using $C=T d S / d T$. Figure 3(a) shows the field dependence of $C / T$ at $T / T_{c}=0.4$ in a $d$-wave superconductor. In the absence of the paramagnetic effect $\left(\alpha_{\text {para }}=0\right)$, the field dependence of $C / T$ has a negative curvature, which coincides with the results shown in refs. 15 and 16 . With increasing $\alpha_{\text {para }}$, however, the sign of the curvature changes from negative to positive. This is more evident in Fig. 3(b), where the same data are plotted against the normalized field $B / H_{c 2}(T)$. This field dependence is consistent with the observation in $\mathrm{CeCoIn}_{5}{ }^{9,11,12}$ It is worth noting that this behavior can be seen irrespective of the structure of the pairing function. Actually, the same behavior is seen for the $s$-wave case, ${ }^{31}$ as shown in the inset of Fig. 3(a). Also we would like to mention that although our result is based on an approximate solution and should be understood as a semi-quantitative one at most, an extensive numerical calculation ${ }^{32}$ using the so-called explosion method supports the conclusion.

To understand the microscopic origin of the behavior $C(B) \propto B^{2}$, it is convenient to relate the magnetization with $C(B)$ through a thermodynamic Maxwell's relation,

$$
\frac{\partial}{\partial B}\left(\frac{C(B)}{T}\right)=\frac{\partial^{2}}{\partial T^{2}}(M(T))
$$

indicating that the $T^{2}$-coefficient of the magnetization gives the slope of $C(B) / T$. Since the magnetization can be divided into the paramagnetic $\left(M_{\text {para }}\right)$ and diamag-

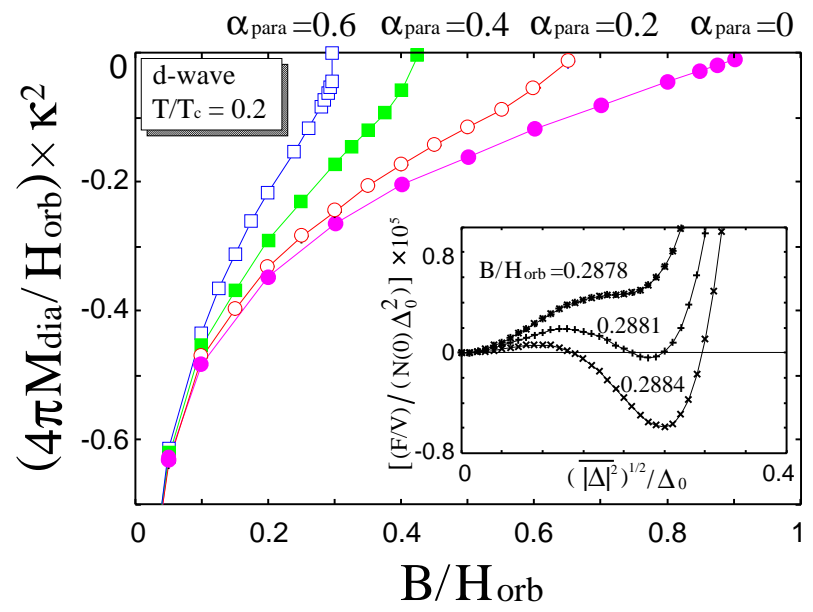

Fig. 2. Field dependence of $M_{\mathrm{dia}}$ at $T / T_{c}=0.2$ in $d$-wave superconductor for $\alpha_{\text {para }}=0,0.2,0.4$, and 0.6. Inset: free energy near $H_{c 2}$ vs pair field strength for $\alpha_{\text {para }}=0.6$. The expression used for the free energy is the same as eq. (4) in ref. 23. netic $\left(M_{\text {dia }}\right)$ parts, we can separately discuss the corresponding contribution to $C(B)$ through the above relationship. Figure 4 shows the temperature dependences of $M_{\text {dia }}$ and $M_{\text {para }}$ for $\alpha_{\text {para }}=0.6$ at several fields. It is easily seen that $M_{\text {para }}$ changes like $T^{2}$ and this coefficient increases with increasing magnetic field, whereas $M_{\text {dia }}$ seems to be almost $T$-linear and has a small $T^{2}$ -
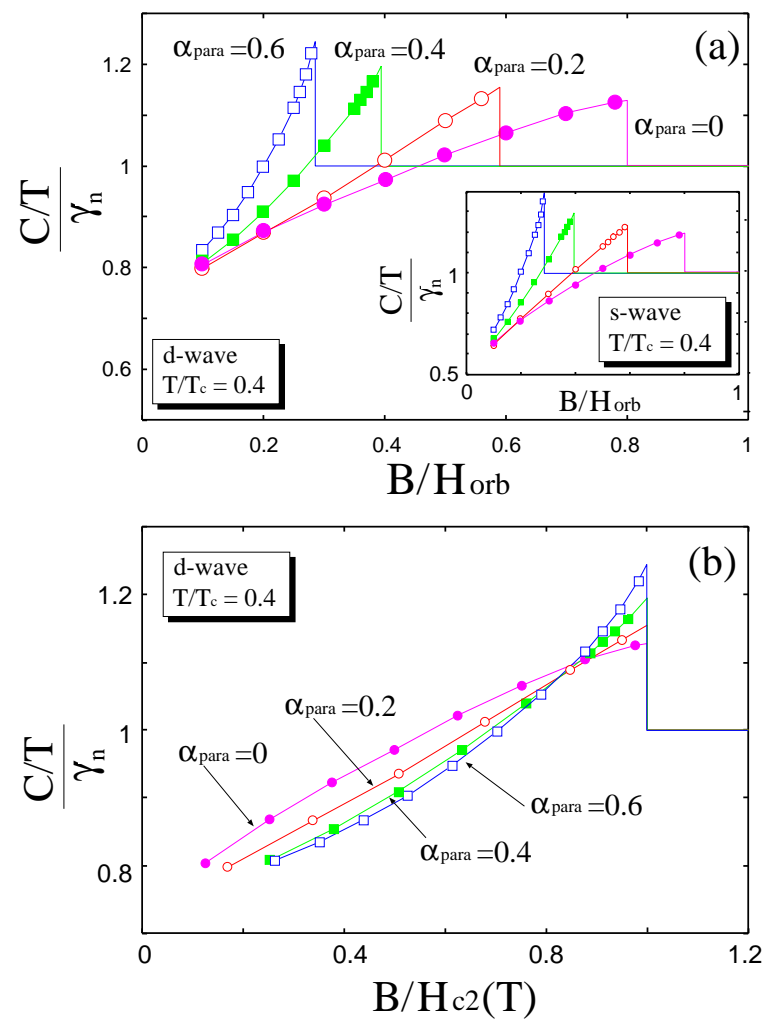

Fig. 3. (a) Field dependence of specific heat at $T / T_{c}=0.4$ for $d$-wave case. Inset: corresponding data for $s$-wave case. Here, $\gamma_{\mathrm{n}}=2 \pi^{2} N(0) / 3$. (b) Data in (a) are plotted as a function of the normalized field $B / H_{c 2}(T)$.

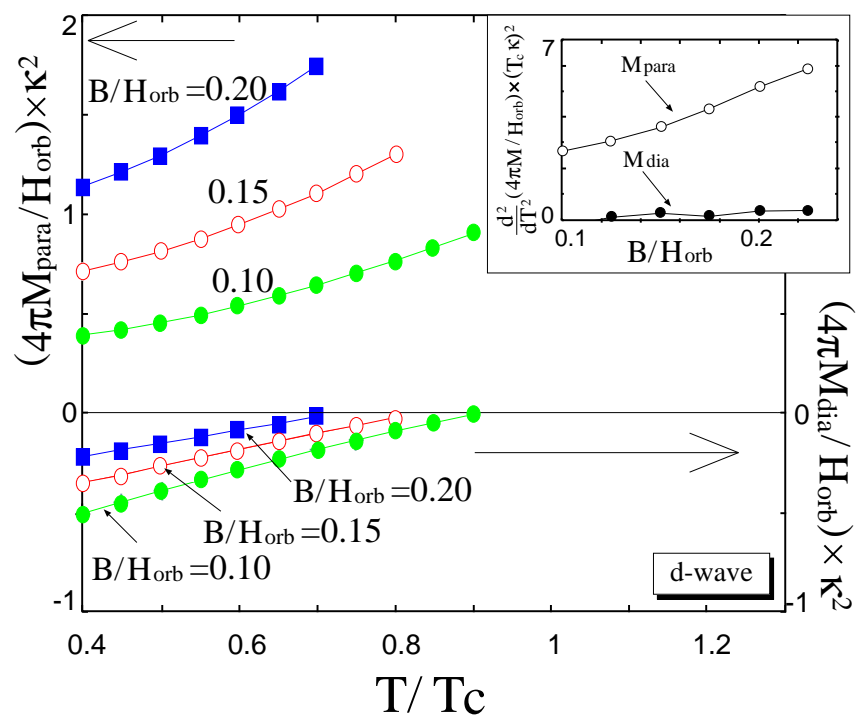

Fig. 4. Temperature dependences of $M_{\text {para }}$ and $M_{\text {dia }}$ for $\alpha_{\text {para }}=$ 0.6 at several fields. Inset: field dependences of $\partial^{2} M_{\text {para }} / \partial T^{2}$ and $\partial^{2} M_{\mathrm{dia}} / \partial T^{2}$ at $T / T_{c}=0.4$ for $\alpha_{\mathrm{para}}=0.6$. 
coefficient. This is clearer in the inset of Fig. 4, where the field dependences of $\partial^{2} M_{\text {para }} / \partial T^{2}$ and $\partial^{2} M_{\text {dia }} / \partial T^{2}$ are shown; $\partial^{2} M_{\text {para }} / \partial T^{2}$ is almost $B$-linear and gives the $B^{2}$-dependence of $C(B)$, while $\partial^{2} M_{\mathrm{dia}} / \partial T^{2}$ is almost field-independent and does not give a large field dependence. Thus, the main source of the behavior $C(B) \propto B^{2}$ is not the vortex contribution but the paramagnetic depairing of the Cooper pair that lowers $H_{c 2}$ considerably. It is interesting to point out that $C(B) / T \propto \sqrt{B}$ for a nodal gap case $(\propto B$ for a gapful case) at low temperatures indicates $\beta(B) \propto 1 / \sqrt{B}(\propto$ const $)$, where $M(T)=M(0)+\alpha(B) T+\beta(B) T^{2}+O\left(T^{3}\right)$. Although there is no accurate measurement for $M(T)$ to check it, the existing data of $\mathrm{YNi}_{2} \mathrm{~B}_{2} \mathrm{C}^{33}$ and $\mathrm{MgB}_{2}{ }^{34}$ seems to support it. It is desirable to confirm this internal consistency for other superconductors.

We briefly discuss the data of $\mathrm{Sr}_{2} \mathrm{RuO}_{4}$, which is believed to be a prime candidate for the triplet pairing. For $\mathbf{H} \perp c, C(B) / T$ shows a strong positive curvature and changes smoothly into a $\sqrt{B}$ behavior as $\mathbf{H}$ rotates towards the $c$-direction ${ }^{35}$ reminiscent of the change with $\alpha_{\text {para }}$ shown in Fig. 3. This may be understandable if we assume either that the $d$-vector in $\mathrm{Sr}_{2} \mathrm{RuO}_{4}$ is strongly locked within the basal plane ${ }^{36}$ or singlet pairing symmetry. Upon rotating the field direction, the relative weight $H_{\text {orb }}$ to the paramagnetic effect $\mu B$, or $\alpha_{\text {para }}$, changes, and $C(B) / T$ should show positive to negative curvatures. Note that ${ }^{37}$ (i) the in-plane magnetization curve exhibits a jump near $H_{c 2}$ at low temperatures, (ii) the Maki parameter $\kappa_{2}$ is an increasing function of temperature, and (iii) $H_{c 2}(\perp c)$ is strongly suppressed; these behaviors are similar to those of $\mathrm{CeCoIn}_{5}$.

In conclusion, we have studied the mixed-state thermodynamics of a superconductor with moderately large paramagnetic effects. Our numerical calculation based on the quasiclassical Eilenberger formalism revealed that with increasing paramagnetic effect, (i) the Maki parameter $\kappa_{2}(T)$ becomes indeed an increasing function of $T$ and (ii) a $C(B) \propto B^{2}$ behavior appears. It can be said that the field dependence of the specific heat and magnetization is a good probe for measuring the strength of paramagnetic effects in type-II materials. Moreover, our demonstration provides a natural explanation for the $C(B) \propto B^{2}$ behavior observed in $\mathrm{CeCoIn}_{5}$ in terms of the paramagnetic effect.

After this work was completed, we were informed ${ }^{38}$ that a $C(B) \propto B^{2}$ behavior is a universal phenomenon for a system on the verge of exhibiting a first-order transition of superconductivity; thus, it can also arise from a strong coupling effect such as antiferromagnetic fluctuations.

We acknowledge fruitful discussions with T. Sakakibara, R. Ikeda, K. Tenya, T. Tayama, and K. Deguchi.

1) C. Petrovic, P. G. Pagliuso, M. F. Hundley, R. Movshovich, J. L. Sarrao, J. D. Thompson, Z. Fisk and P. Monthoux: J. Phys.: Condens. Matter 13 (2001) L337.
2) K. Izawa, H. Yamaguchi, Y. Matsuda, H. Shishido, R. Settai and Y. Onuki: Phys. Rev. Lett. 87 (2001) 057002.

3) A. Bianchi, R. Movshovich, N. Oeschler, P. Gegenwart, F. Steglich, J. D. Thompson, P. G. Pagliuso and J. L. Sarrao: Phys. Rev. Lett. 89 (2002) 137002.

4) T. Tayama, A. Harita, T. Sakakibara, Y. Haga, H. Shishido, R. Settai and Y. Onuki: Phys. Rev. B 65 (2002) 180504(R).

5) H. Adachi and R. Ikeda: Phys. Rev. B. 68 (2003) 184510.

6) H. A. Radovan, N. A. Fortune, T. P. Murphy, S. T. Hannahs, E. C. Palm, S. W. Tozer and D. Hall: Nature (London) 425 (2003) 51.

7) A. Bianchi, R. Movshovich, C. Capan, P. G. Pagliuso and J. L. Sarrao: Phys. Rev. Lett. 91 (2003) 187004.

8) T. Watanabe, Y. Kasahara, K. Izawa, T. Sakakibara, Y. Matsuda, C. J. van der Beek, T. Hanaguri, H. Shishido, R. Settai and Y. Onuki: Phys. Rev. B 70 (2004) 020506(R).

9) H. Aoki, T. Sakakibara, H. Shishido, R. Settai, Y. Onuki, P. Miranović and K. Machida: J. Phys.: Condens. Matter 16 (2004) L13.

10) R. Ikeda and H. Adachi: Phys. Rev. B 69 (2004) 212506.

11) S. Ikeda, H. Shishido, M. Nakashima, R. Settai, D. Aoki, Y. Haga, H. Harima, Y. Aoki, T. Namiki, H. Sato and Y. Onuki: J. Phys. Soc. Jpn 70 (2001) 2248.

12) K. Deguchi: private communication.

13) G. E. Volovik: JETP Lett. 58 (1993) 469.

14) C. Caroli, P. G. de Gennes and J. Matricon: Phys. Lett. 9 (1964) 307.

15) M. Ichioka, A. Hasegawa and K. Machida: Phys. Rev. B 59 (1999) 8902

16) N. Nakai, P. Miranović, M. Ichioka and K. Machida: Phys. Rev. B 70 (2004) 100503(R).

17) A. P. Ramirez, C. M. Varma, Z. Fisk and J. L. Smith: Phil. Mag. B 79 (1999) 111.

18) A. L. Fetter and P. C. Hohenberg: Superconductivity, ed. P. D. Parks (Dekker, New York, 1969) Vol. 2, p. 881.

19) I. Vekhter, P. J. Hirschfeld and E. J. Nicol: Phys. Rev. B 64 (2001) 064513.

20) N. D. Whelan and J. P. Carbotte: Phys. Rev. B 62 (2000) 14511.

21) G. Eilenberger: Z. Phys. 214 (1968) 195.

22) H. Adachi, P. Miranović, M. Ichioka and K. Machida: Phys. Rev. Lett. 94 (2005) 067007.

23) T. Kita: Phys. Rev. B 69 (2004) 144507.

24) U. Klein: Phys. Rev. B 69 (2004) 134518.

25) W. Pesch: Z. Phys. B 21 (1975) 263.

26) A. Houghton and I. Vekhter: Phys. Rev. B 57 (1998) 10831; T. Dahm, S. Graser, C. Iniotakis and N. Schopohl: Phys. Rev. B 66 (2002) 144515; H. Kusunose: Phys. Rev. B 70 (2004) 054509; M. Udagawa, Y. Yanase and M. Ogata: Phys. Rev. B 70 (2004) 184515.

27) K. Maki: Phys. Rev. 148 (1966) 362.

28) M. Houzet and A. Buzdin: Europhys. Lett. 50 (2000) 375.

29) G. Eilenberger: Phys. Rev. 153 (1967) 584.

30) T. Kita: Phys. Rev. B 68 (2003) 184503.

31) Our calculation was performed at relatively high temperatures, covering the behavior that depends on the pairing symmetry. In a zero-temperature limit, we observe a $C \propto \sqrt{B}$-like behavior $^{32}$ at a low field region for a line node case.

32) M. Ichioka: private communication.

33) K. J. Song, J. R. Thompson, M. Yethiraj, D. K. Christen, C. V. Tomy and D. McK. Paul: Phys. Rev. B. 59 (1999) 6620(R).

34) B. Kang, H.-J. Kim, M.-S. Park, K.-H. Kim and S.-I. Lee: Phys. Rev. B. 69 (2004) 144514.

35) K. Deguchi, Z. Q. Mao and Y. Maeno: J. Phys. Soc. Jpn. 73 (2004) 1313.

36) C. H. Choi and J. A. Sauls: Phys. Rev. Lett. 66 (1991) 484.

37) K. Tenya: private communication.

38) R. Ikeda: private communication. 